\title{
Endoscopic Management of Gastrointestinal Leaks and Bleeding with the Over-the-Scope Clip: A Prospective Study
}

\author{
Mahesh Kumar Goenka ${ }^{1}$, Vijay Kumar Rai ${ }^{1}$, Usha Goenka ${ }^{2}$ and Indrajit Kumar Tiwary ${ }^{1}$ \\ ${ }^{1}$ Institute of Gastrosciences, ${ }^{2}$ Department of Imaging and Interventional Radiology, Apollo Gleneagles Hospitals, Kolkata, India
}

Background/Aims: The over-the-scope clip (OTSC) is a device used for endoscopic closure of perforations, leaks and fistulas, and for endoscopic hemostasis. To evaluate the clinical effectiveness and safety of OTSC.

Methods: Between October 2013 and November 2015, 12 patients underwent OTSC placement by an experienced endoscopist. OTSC was used for the closure of gastrointestinal (GI) leaks and fistula in six patients, three of which were iatrogenic (esophageal, gastric, and duodenal) and three of which were inflammatory. In six patients, OTSC was used for hemostasis of non-variceal upper GI bleeding. Endoscopic tattooing using India ink was used to assist the accurate placement of the clip.

Results: All subjects except one with a colonic defect experienced immediate technical success as well as long-term clinical success, during a mean follow-up of 6 weeks. Only one clip was required to close each of the GI defects and to achieve hemostasis in all patients. There were no misfirings or complications of clips. The procedure was well tolerated, and patients were hospitalized for an average of 8 days (range, 3 to 10). Antiplatelet therapy was continued in patients with GI bleeding.

Conclusions: In our experience, OTSC was safe and effective for the closure of GI defect and to achieve hemostasis of non-variceal GI bleeding. Clin Endosc 2017;50:58-63

Key Words: Ovesco; Over-the-scope clip; Fistula closure; Gastrointestinal hemorrhage

\section{INTRODUCTION}

Gastrointestinal (GI) leaks and fistulae, both iatrogenic and inflammatory, have been traditionally treated at endoscopy with through-the-scope clips (TTSCs), self-expandable metal stents, and glues. ${ }^{1-3}$ However, these modalities have limitations, such as stent migration, incomplete closure of the defect, or difficulty in stent removal. ${ }^{4,5}$ Conventional endoscopic treatment of GI bleeding has a failure rate of approximately 10\%, related to the patient's age, severity of bleeding, and comorbidities. ${ }^{6}$ The application of TTSCs has some drawbacks in more

Received: January 23, 2016 Revised: April 11, 2016

Accepted: April 17, 2016

Correspondence: Vijay Kumar Rai

Institute of Gastrosciences, Apollo Gleneagles Hospitals, 58 Canal Circular Road, Kolkata 700054, India

Tel: +91-33-2320-3040, Fax: +91-33-2320-5218, E-mail: vijayrai80@gmail.com

(cc) This is an Open Access article distributed under the terms of the Creative Commons Attribution Non-Commercial License (http://creativecommons.org/ licenses/by-nc/3.0) which permits unrestricted non-commercial use, distribution, and reproduction in any medium, provided the original work is properly cited. difficult, bleeding lesions because of the pointed ends of the clips. Multiple clips may have to be applied in some of these lesions. Recent studies have shown that over-the-scope clips (OTSCs; Ovesco, Tübingen, Germany) allow effective sealing of large defects as well as hemostasis of complex bleeding lesions. ${ }^{7,8}$ The OTSC system is currently available in our region, but reported experience with the OTSC is very scarce. This study aimed to evaluate the safety and efficacy of OTSC in the closure of leaks and in lesions with ongoing, difficult to control bleeding.

\section{MATERIALS AND METHODS}

\section{Patient selection}

We performed a single-center prospective study on the use of the OTSC system in patients with evidence of a GI leak or fistula and ongoing refractory non-variceal GI bleeding. Twelve consecutive patients who underwent OTSC placement over 2 years between October 2013 and November 2015 were 
evaluated. OTSCs were used only in those GI bleeding cases in which conventional endoscopic therapy (TTSC clip, thermal coagulation, and/or injection of adrenaline) had failed. All interventions were performed at the Institute of Gastrosciences in our hospital.

\section{Ovesco clip system}

The OTSC (Ovesco) is made up of biocompatible nitinol, having a high degree of elasticity and memory which allows it to return to its original shape after deployment. The clip is marketed preloaded into a transparent applicator cap in a curved state before deployment. Available clip sizes range from 11 to $14 \mathrm{~mm}$. Three versions of OTSCs are available: a blunt atraumatic type, one with pointed traumatic edges, and a clip for gastric wall closure with features of both the traumatic and atraumatic OTSC. The twin grasper and anchors are accessories used to approximate the tissue margins of large or fibrotic gaping defects and to pull these into the transparent cap before releasing the clip.

The OTSC system was attached to the tip of a gastroscope (Olympus GIF-HQ190; diameter of $9.9 \mathrm{~mm}$; working channel of $2.8 \mathrm{~mm}$; Olympus, Tokyo, Japan) or a colonoscope (Olympus CF-HQ190 ; diameter of $13.2 \mathrm{~mm}$; working channel of 3.7 $\mathrm{mm}$; Olympus), in a manner almost similar to the band ligation device. A double-channel therapeutic gastroscope (Olym- pus GIF-2TH180; Olympus) was used when accessories such as the twin grasper or anchor were to be used. The fistula and perforations were marked by injecting India ink to accurately localize the site while deploying the OTSC.

The clip was deployed by rotating a handle attached to the working channel of the endoscope, a technique similar to the one performed during variceal ligation at endoscopy. All procedures were conducted under sedation with intravenous propofol. All endoscopic procedures were carried out by a single experienced endoscopist. The Ovesco system was described to patients, and informed consent was obtained from them.

\section{Follow-up}

Patients were followed up for at least 4 weeks after the endoscopic intervention. In GI defect cases, technical success was determined through endoscopy and/or radiography, and clinical success was defined as a persistent closure of the GI defect with clinical improvement. In GI bleed cases, technical success was defined as immediate hemostasis with hemodynamic stability, and clinical success was defined as stable hemoglobin levels during follow-up with no signs of re-bleeding.
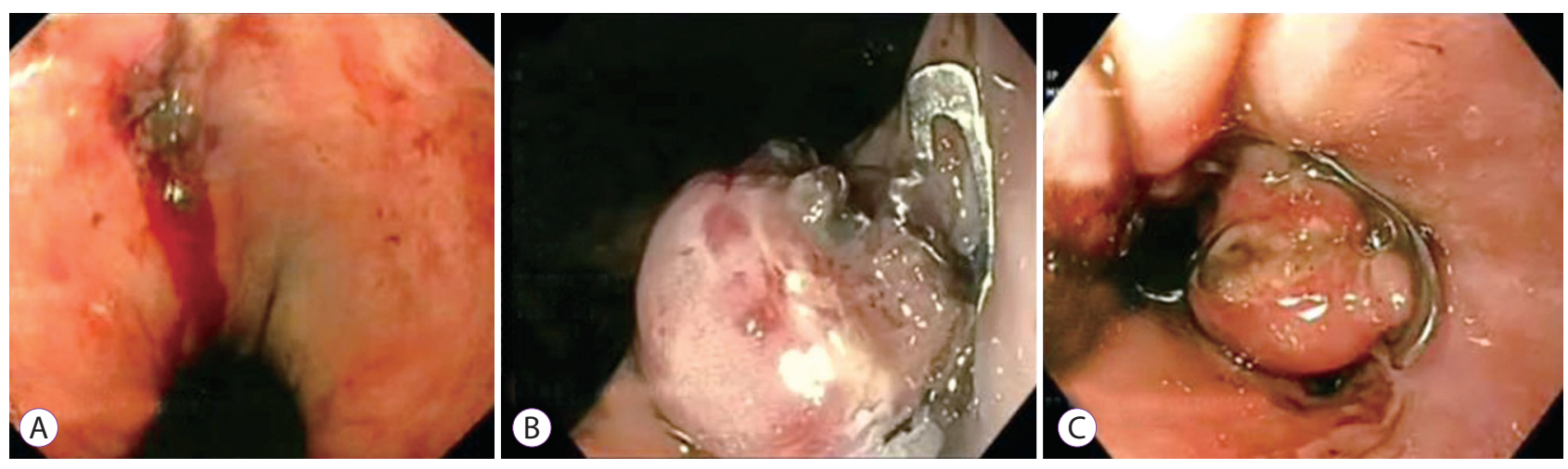

Fig. 1. (A) Bleeding from Mallory-Weiss tear. (B) Ovesco clip deployed to control the bleeding. (C) Clip in situ after 2 days.
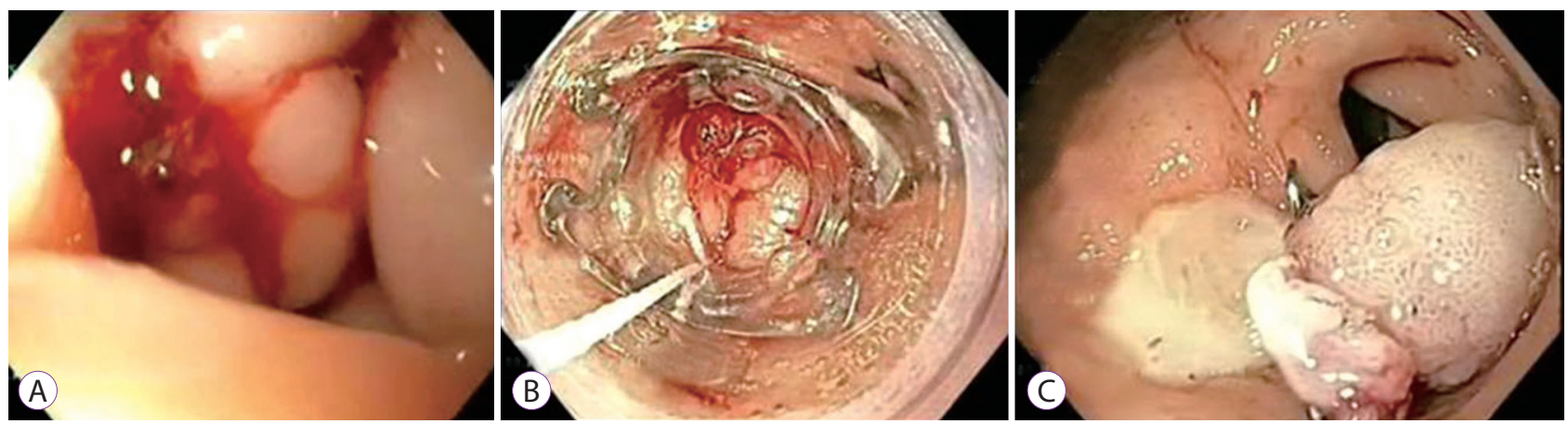

Fig. 2. (A) Forrest class $1 \mathrm{~b}$ bleeding from a duodenal ulcer. (B) Ovesco clip deployed to close the bleeding point. (C) Clip in situ after 2 days with no rebleeding. 


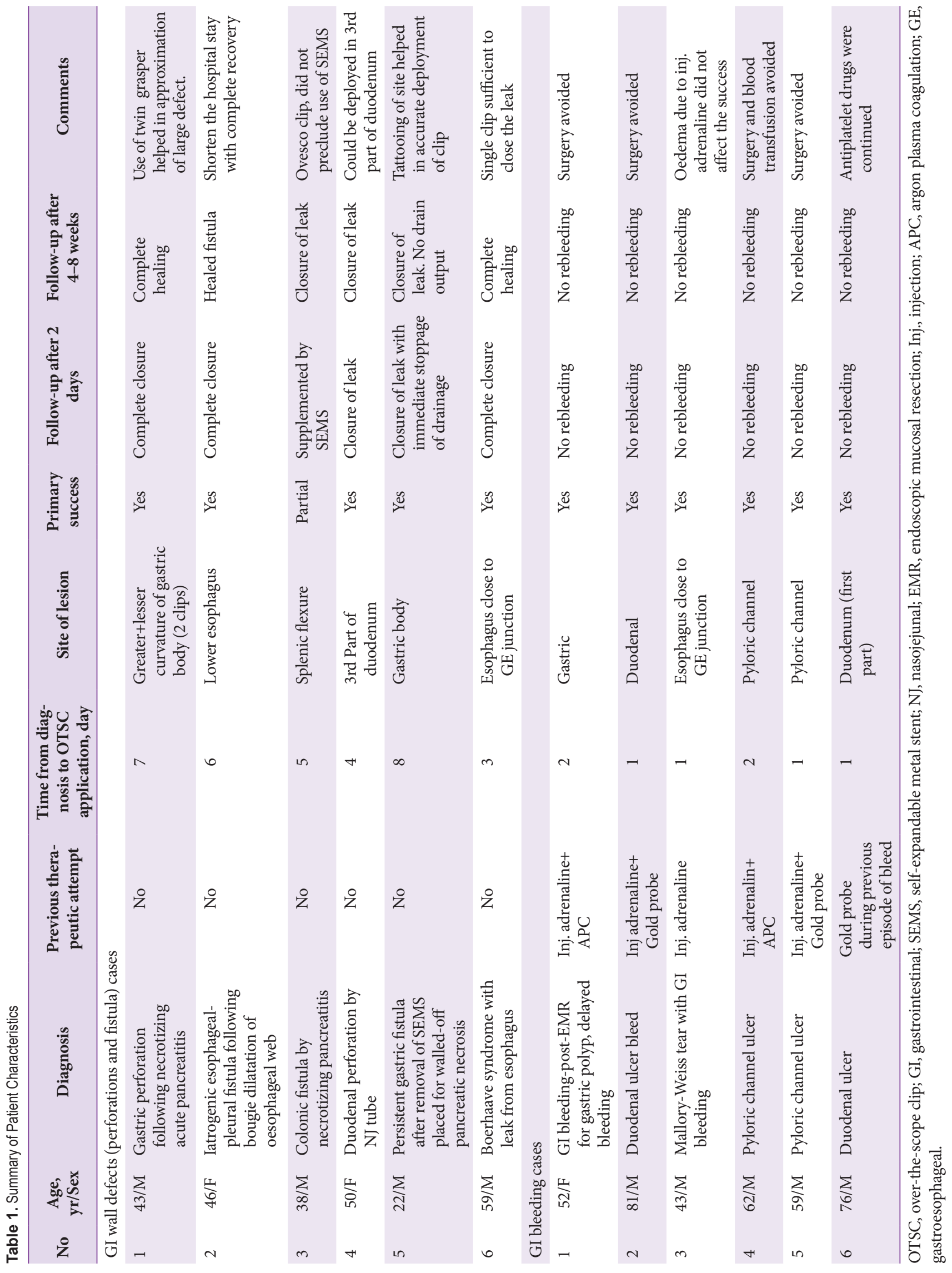




\section{RESULTS}

We used 13 OTSCs in 12 different patients (nine men, three women), aged between 22 and 81 years. A summary of patients' age, sex, diagnosis, indications for OTSC application, time to diagnosis and clip application, and technical and clinical success is shown in Table 1. Patients were hospitalized for an average of 8 days (range, 3 to 10) and followed up for a mean of 5 to 18 weeks. Four OTSC clips were applied to the stomach, three to the esophagus, one to the colon, and five to the pyloric channel or duodenum. In six cases, the clip was applied to close the GI wall defect and in another six cases, the clip was applied to control major refractory non-variceal GI bleeding.

Out of six cases of GI bleeding, two had bleeding from the duodenum, two from the pyloric channel, one from the stomach, and one from the lower esophagus close to the gastro-esophageal junction (Fig. 1). Three cases had Forrest type 1a ulcer bleeding, while three had Forrest type $1 \mathrm{~b}$ bleeding (Fig. 2). Three patients had severe bleeding with a fall in hemoglobin to below $5 \mathrm{~g} / \mathrm{dL}$ with hypovolemic shock. All cases were initially managed with adrenaline injections and gold probe or argon plasma coagulation application, and were later managed with OTSC when these conventional modalities failed. One patient (Table 1; No. 6) was on antiplatelet drugs, after placement of a drug-eluting coronary stent, which were continued after hemostasis was achieved following Ovesco clip application. Clinical and technical success was achieved in all six bleeding cases, accounting to a $100 \%$ overall success with no evidence of further bleeding.

Of the GI wall defect cases, three were of iatrogenic perforation with less than a 1-week interval between detection of defects and clip application. Of these three cases, one was of a perforation of the third part of the duodenum due to a nasojejunal tube (Fig. 3), the second was of an esophageal perforation due to bougie dilatation of an esophageal web (Fig. 4), and the third one was of a persistent gastric fistula following removal of a lumen opposing metal stent placed for walled-off necrosis of the pancreas. All cases were managed successfully with OTSC clip without any recurrence. The remaining three cases of GI wall defects were fistulae due to Boerhaave syndrome (one case) and necrotizing pancreatitis (two cases, one case in the stomach and the other in the colon). The patient with fistulas in the stomach due to necrotizing pancreatitis had two fistulae, one each on the lesser and greater curvature. Two clips were deployed successfully to close both defects in
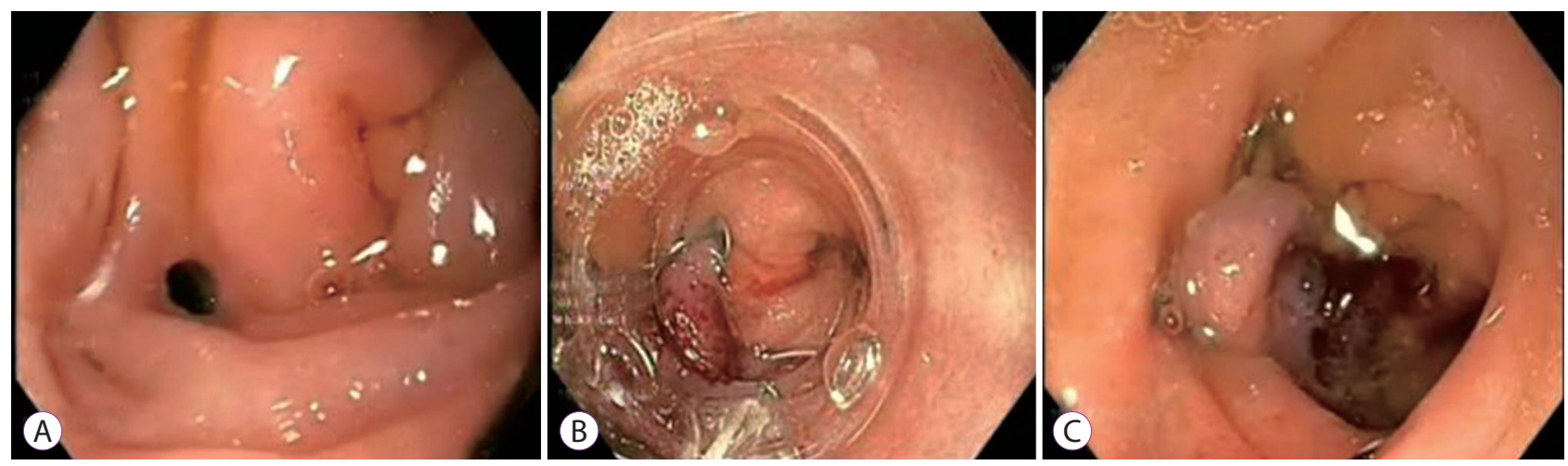

Fig. 3. (A) Endoscopic view of an iatrogenic nasojejunal tube-related duodenal perforation. (B) Closure by Ovesco clip. (C) The site 7 days after application of the clip.
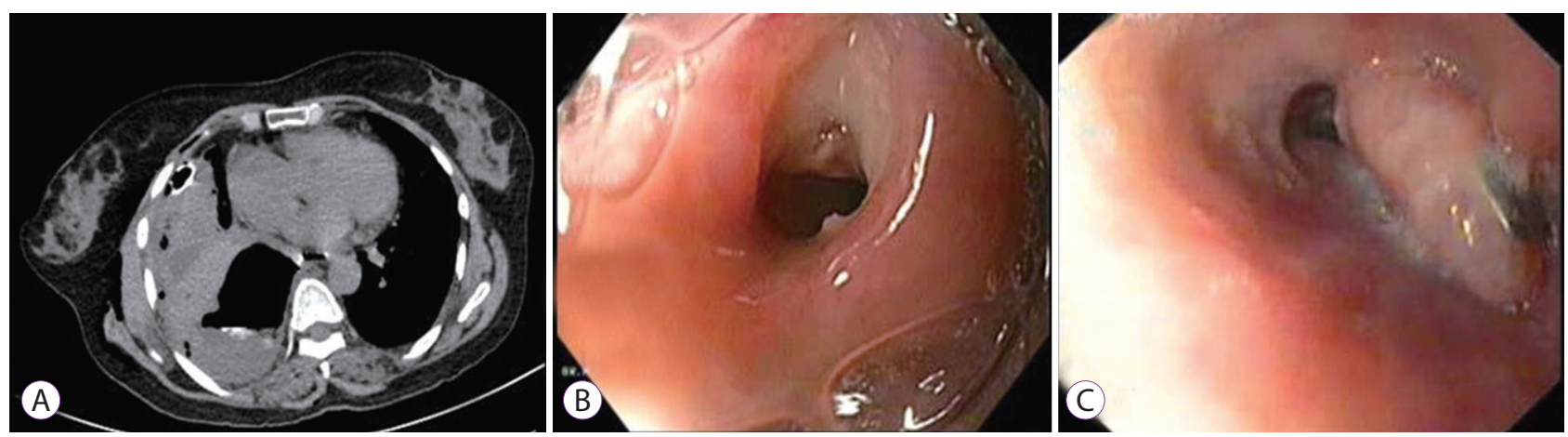

Fig. 4. latrogenic esophageal-pleural fistula following bougie dilatation. (A) Fistula shown on computed tomography image. (B) Endoscopic image of esophageal opening of fistula. (C) Successful closure by Ovesco clip. 
the same session. Twin grasper and anchors were used in this case due to the large size of the defect. The colonic fistula was closed only partially with OTSC. Technical failure of closure of the colonic fistula was detected using computed tomography scan a day after the clip application, and was managed in the additional endoscopic procedure by deploying a fully covered colonic nitinol metal stent. This led to a complete closure of the fistula without any recurrence, even after removal of the stent at 6 weeks. No complications related to the OTSC or to the clip application technique were noted. Thus in six cases of GI wall defect, technical as well as long-term clinical success were achieved in five cases accounting to an $83.3 \%$ success rate in the GI wall defect group. The overall success rate combining GI bleeding and GI wall defect cases was $91.7 \%$. No patients required surgery after Ovesco clip application, and the mean hospital stay was 8 days (range, 3 to 10). Four patients had the clip in situ at 8 weeks of follow-up.

\section{DISCUSSION}

The OTSC system is a novel procedure that allows the sealing of GI defects in the form of fistulae, perforations, and leaks, and may also control severe bleeding from lesions in the GI tract. This clip has advantages over traditional TTSCs in terms of depth of tissue and size of lesion grasped, ease of application, and closure power, as shown in many studies. ${ }^{7-9}$ It is quick and easy to use, particularly for endoscopists with expertise in variceal ligation. In this study, we noted great ease and success in sealing fistulae or perforations as well as controlling bleeding with the use of OTSC. The morbidity and mortality associated with surgery, which was an available alternative, were avoided with OTSC. Although this study was a small case series and had a heterogeneous population, the results seem encouraging.

In the first multicenter series of OTSC in 45 patients for various indications such as closure of chronic fistulas, perforation, anastomotic leaks after esophagectomy, hemostasis, leakage from the gallbladder, and a defect due to subepithelial mass resection, the overall success rate was $71 \% .^{10}$ The success was decided by the indication, being $100 \%$ for hemostasis, $75 \%$ for perforation, and $65 \%$ for fistula closure. ${ }^{10}$ In the largest retrospective multicenter review involving 188 patients undergoing OTSC placement for GI defects, longterm success was seen in $60.2 \%$ during a median follow-up of 146 days. ${ }^{11}$ Nearly $60 \%$ of the patients had OTSC placed as primary or initial therapy. The low success rate in this series was probably due to the inclusion of a significant proportion of patients having the clip placed more than a month after the diagnosis of the fistula. It was demonstrated in this study that the OTSC system had limited efficacy for chronic fistulae of more than a 4-week duration compared to more recent defects. ${ }^{11}$ Important determinants of long-term success were type of defect, acute injury, and OTSC use as a primary rather than a rescue therapy. In our series, all six patients presented with acute GI leaks of less than 10 days duration. We did not experience many of the difficulties described in other case series, and achieved success in all but one case. In all cases of GI defect, we marked the site of the defect by tattooing with India ink, which seems to have helped us in accurately deploying the clip and also shortened the procedure time. We used the anchoring device with non-sharp edges successfully in two fistulae because of large defect sizes of around $2 \mathrm{~cm}$. Persistent closure was achieved in the present study in both iatrogenic as well as spontaneous GI defects. Placement of hemostatic TTSC clips leads to superficial mucosal closure, which does not include the muscular layer in cases of perforation. ${ }^{12}$ OTSC not only allows the closure of large defects measuring as much as $27 \mathrm{~mm}$, but also includes the muscular layer of defects, as shown in previous studies. ${ }^{13,14}$

In the present series, the final success rate was $91.7 \%$, which is similar to the success rate achieved by Manta et al. ${ }^{15}$ who reported a success rate of $90 \%$ in 12 patients. Parodi et al. ${ }^{16}$ had a success rate of $80 \%$ in a small series of 10 patients with GI fistulae and perforations in different locations. Experience with clip application in the colon is very limited, and we had one partial failure in a patient with a colonic fistula. The reason for this is unknown, but it may be due to poor visualization and angulation of the defect while applying the clip.

In GI bleeding, the OTSC has been shown to have less impact than it has in postoperative fistulae. Large bleeding ulcers remain a therapeutic challenge. In all six GI bleeding cases we managed, we achieved 100\% technical and clinical success, probably due to proper case selection and clear visualization of the bleeding point. A $100 \%$ success rate in a small case series with a long-term success of more than $90 \%$ has been reported previously. ${ }^{15,16}$ One of our patients with an ulcer bleed (Table 1; No. 6) was taking antiplatelet drugs after coronary stenting. This patient had a previous episode of ulcer bleeding managed by coagulation and temporary stoppage of anti-platelet drugs. Recurrence of bleeding from the ulcer was managed by the Ovesco clip without interruption of the anti-platelet medication. Secure hemostasis of the bleeding lesion may allow continuance of antiplatelet drugs in patients with high cardiovascular risk. In the present series there were no immediate or late complications associated with OTSC placement. Severe adverse events such as complete lumen closure secondary to clip misplace- 
ment and mucosal injury caused by the pointed teeth of the OTSC during insertion have been described rarely in the literature. $^{16,17}$ Therefore, caution should be exercised when inserting the OTSC into a relatively narrow area, such as the cricopharynx or the pylorus. The main disadvantage of the Ovesco clip is its cost and the need for reintroduction of the scope to deploy the clips after noting the lesion at endoscopy. However, if we compare the length of hospital stay, the need for blood transfusions and surgery, and the related morbidity and mortality, it may be cost effective.

To our knowledge, no case series from our region has reported on the clinical use of the OTSC in GI bleeding, closure of fistulae after the removal of metal stents, closure of gastric wall defects in necrotizing pancreatitis, or closure of esophageal-pleural fistula.

In conclusion, the major benefits of the OTSC are its speed and ease of deployment, and the persistent sealing of leaks and fistulae of the GI tract. Its use appears to be most effective in GI defects of less than 1-week duration. OTSCs may also be helpful in selected patients with uncontrolled non-variceal bleeding and large GI bleeding sites. As shown in one of our patients, antiplatelet drugs, which may be essential in certain situations such as recently deployed drug-eluting coronary stents, may be continued after successful placement of OTSC clips. Application of the clip seems to be safe and less invasive. We have used tattooing to mark the leak site, and feel that this modification can help in accurate placement and success of OTSC. Further refinement of the OTSC technique is, however, needed for closing larger, chronic, and more complex defects. Large prospective studies are needed to determine the criteria for recognizing the best suitable lesions for this clipping device.

\section{Conflicts of Interest}

The authors have no financial conflicts of interest.

\section{REFERENCES}

1. Schecter WP, Hirshberg A, Chang DS, et al. Enteric fistulas: principles of management. J Am Coll Surg 2009;209:484-491.
2. Cho SB, Lee WS, Joo YE, et al. Therapeutic options for iatrogenic colon perforation: feasibility of endoscopic clip closure and predictors of the need for early surgery. Surg Endosc 2012;26:473-479.

3. Rábago LR, Ventosa N, Castro JL, Marco J, Herrera N, Gea F. Endoscopic treatment of postoperative fistulas resistant to conservative management using biological fibrin glue. Endoscopy 2002;34:632-638.

4. Bège T, Emungania O, Vitton V, et al. An endoscopic strategy for management of anastomotic complications from bariatric surgery: a prospective study. Gastrointest Endosc 2011;73:238-244.

5. Amrani L, Ménard C, Berdah S, et al. From iatrogenic digestive perforation to complete anastomotic disunion: endoscopic stenting as a new concept of "stent-guided regeneration and re-epithelialization". Gastrointest Endosc 2009;69:1282-1287.

6. Rockall TA, Logan RF, Devlin HB, Northfield TC. Risk assessment after acute upper gastrointestinal haemorrhage. Gut 1996;38:316-321.

7. Kirschniak A, Kratt T, Stüker D, Braun A, Schurr MO, Königsrainer A. A new endoscopic over-the-scope clip system for treatment of lesions and bleeding in the GI tract: first clinical experiences. Gastrointest Endosc 2007;66:162-167.

8. Repici A, Arezzo A, De Caro G, et al. Clinical experience with a new endoscopic over-the-scope clip system for use in the GI tract. Dig Liver Dis 2009;41:406-410.

9. Kirschniak A, Traub F, Kueper MA, Stüker D, Königsrainer A, Kratt T. Endoscopic treatment of gastric perforation caused by acute necrotizing pancreatitis using over-the-scope clips: a case report. Endoscopy 2007;39:1100-1102.

10. Baron TH, Song LM, Ross A, Tokar JL, Irani S, Kozarek RA. Use of an over-the-scope clipping device: multicenter retrospective results of the first U.S. experience (with videos). Gastrointest Endosc 2012;76:202-208.

11. Haito-Chavez Y, Law JK, Kratt T, et al. International multicenter experience with an over-the-scope clipping device for endoscopic management of GI defects (with video). Gastrointest Endosc 2014;80:610-622.

12. von Renteln D, Schmidt A, Vassiliou MC, Rudolph HU, Gieselmann $\mathrm{M}$, Caca K. Endoscopic closure of large colonic perforations using an over-the-scope clip: a randomized controlled porcine study. Endoscopy 2009;41:481-486.

13. Schurr MO, Hartmann C, Kirschniak A, Ho CN, Fleisch C, Buess G. Experimental study on a new method for colonoscopic closure of large-bowel perforations with the OTSC clip. Biomed Tech (Berl) 2008;53:45-51.

14. Pohl J, Borgulya M, Lorenz D, Ell C. Endoscopic closure of postoperative esophageal leaks with a novel over-the-scope clip system. Endoscopy 2010;42:757-759.

15. Manta R, Manno M, Bertani H, et al. Endoscopic treatment of gastrointestinal fistulas using an over-the-scope clip (OTSC) device: case series from a tertiary referral center. Endoscopy 2011;43:545-548.

16. Parodi A, Repici A, Pedroni A, Blanchi S, Conio M. Endoscopic management of GI perforations with a new over-the-scope clip device (with videos). Gastrointest Endosc 2010;72:881-886.

17. Nishiyama N, Mori H, Kobara H, et al. Efficacy and safety of over-thescope clip: including complications after endoscopic submucosal dissection. World J Gastroenterol 2013;19:2752-2760. 